PDES, SUBMANIFOLDS AND

AFFINE DIFFERENTIAL GEOMETRY

BANACH CENTER PUBLICATIONS, VOLUME 69

INSTITUTE OF MATHEMATICS

POLISH ACADEMY OF SCIENCES

WARSZAWA 2005

\title{
BÄCKLUND TRANSFORMS OF CONFORMAL MAPS INTO THE 4-SPHERE
}

\author{
K. LESCHKE and F. PEDIT \\ Department of Mathematics, University of Massachusetts \\ Amherst, MA 01003, U.S.A. \\ E-mail: leschke@gang.umass.edu,franz@gang.umass.edu
}

1. Introduction. Transformations which preserve special surface classes in 3- and 4space play an important role in surface geometry. One of the motivations to study those transformations comes from the fact that they allow one to construct more complicated surfaces from given simple surfaces. Historical examples include the Bäcklund transformation on surfaces of constant Gaussian curvature [Bia80] and the Darboux transformation on isothermic surfaces [Dar99]. More recently, also a Bäcklund transformation on Willmore surfaces has been studied $\left[\mathrm{BFL}^{+} 02\right]$.

In this paper we consider a general Bäcklund transformation on conformal surfaces $f: M \rightarrow S^{4}$ where $M$ is a Riemann surface. This will allow us to explicitly construct new conformal immersions of a given Riemann surface into 4-space from a given one by solving abelian integrals.

Since conformal surface theory is Möbius invariant, we view the 4-sphere as the quaternionic projective line $\mathbb{H P}^{1}$ on which the group of orientation preserving Möbius transformations acts by $\mathrm{GL}(2, \mathbb{H})$. In this framework conformal maps $f: M \rightarrow S^{4}$ are the (quaternionic) holomorphic maps $f: M \rightarrow \mathbb{H P}^{1}$ as introduced in [FLPP01]. In particular, as in complex holomorphic geometry, holomorphic maps correspond via the Kodaira correspondence to holomorphic line bundles together with a 2-dimensional space of holomorphic sections. More precisely, a conformal map $f: M \rightarrow \mathbb{H}^{1}$ induces the quaternionic line bundle $L=f^{*} \mathcal{T}$ where $\mathcal{T} \rightarrow \mathbb{H} \mathbb{P}^{1}$ is the tautological line bundle. The restrictions of linear forms on $\mathbb{H}^{2}$ to $L$ give the 2-dimensional subspace $H \subset H^{0}\left(L^{-1}\right)$ of holomorphic sections of the dual line bundle $L^{-1}$. A "point at infinity" in $\mathbb{H} \mathbb{P}^{1}$ not on the surface $f$, i.e., a linear form $\alpha$ on $\mathbb{H}^{2}$ so that $\left.\alpha\right|_{L} \in H^{0}\left(L^{-1}\right)$ has no zeros, determines a holomorphic

2000 Mathematics Subject Classification: Primary 53C42; Secondary 53Axx.

Key words and phrases: conformal maps, Bäcklund transformation, Willmore surfaces, holomorphic curves.

The paper is in final form and no version of it will be published elsewhere. 
structure on $L$. Then a choice of a 2-dimensional subspace $\tilde{H} \subset H^{0}(L)$ of holomorphic sections of $L$ yields - via the Kodaira embedding — a conformal map $\tilde{f}: M \rightarrow \mathbb{H} \mathbb{P}^{1}$ which we call a Bäcklund transform of $f$. For the special case of Willmore surfaces this construction coincides with the Bäcklund transformation introduced in $\left[\mathrm{BFL}^{+} 02\right]$.

A similar procedure, replacing the point at $\infty$ by a hyperplane at $\infty$, can be carried out for (quaternionic) holomorphic curves $f: M \rightarrow \mathbb{H} \mathbb{P}^{n}$. Depending on the dimension $m$ of $H^{0}(L)$, which for compact $M$ can be estimated by the Riemann-Roch relation [FLPP01] in terms of the genus of $M$ and the degree of $L$, we obtain Bäcklund transforms $\tilde{f}: M \rightarrow \mathbb{H} \mathbb{P}^{l}$ of $f$ for $l \leq m$. Locally on $M$ the dimension of $H^{0}(L)$ is infinite and we obtain local Bäcklund transforms $\tilde{f}: M \rightarrow \mathbb{H}^{l}$ for any $l \in \mathbb{N}$. Since the coordinates $f_{i}: M \rightarrow \mathbb{R}^{4}$ of $f=\left[f_{0}: \ldots: f_{n}\right]$ are all conformal maps this construction gives rise to families of new conformal maps of $M$ into $S^{4}$ from the given holomorphic curve $f$.

A Bäcklund transform $\tilde{f}$ of a holomorphic curve $f: M \rightarrow \mathbb{H} \mathbb{P}^{n}$ depends on the choice of a hyperplane at $\infty$ and a subspace $\tilde{H} \subset H^{0}(L)$ of holomorphic sections of $L$. The latter can be constructed by integrating holomorphic $L$-valued forms $\omega \in H^{0}(K L)$ and we obtain Bäcklund transforms from abelian integrals. This motivates to some extent why we call our construction a Bäcklund transformation rather than a Darboux transformation: for those one usually has to solve Ricatti-type equations.

Geometrically, the abelian integral of $\omega \in H^{0}(K L)$ will have periods and the Bäcklund transform $\tilde{f}$ will only be defined on the universal cover $\tilde{M}$ of $M$. But in the case when $M=T^{2}$ is 2-torus these periods can be closed and we obtain closed Bäcklund transforms $\tilde{f}: T^{2} \rightarrow S^{4}$ from a holomorphic curve $f: T^{2} \rightarrow \mathbb{H}^{n}$.

Given a holomorphic curve $f: M \rightarrow \mathbb{H}^{n}$ the integral of an $L$-valued holomorphic form $\omega \in H^{0}(K L)$ also defines an enveloping curve $\hat{f}: \tilde{M} \rightarrow \mathbb{H}^{n+1}$ of $f$ as discussed in [LP03]. We show that the Bäcklund transform $\tilde{f}$ of $f$ is obtained from a suitable projection of the enveloping curve $\hat{f}$. This gives an interpretation of the Bäcklund transform in terms of fundamental geometric constructions.

We conclude our paper with an application of the Bäcklund transform to constrained Willmore surfaces $f: M \rightarrow S^{4}$. In this situation the bundle $K L$ has a canonical family of holomorphic sections $\omega \in H^{0}(K L)$ whose corresponding Bäcklund transforms are again constrained Willmore. As already mentioned, if $M=T^{2}$ is a 2-torus, the Bäcklund transform $\tilde{f}: T^{2} \rightarrow S^{4}$ has no periods and we can construct new constrained Willmore tori from given ones.

2. Bäcklund transforms of holomorphic curves. We first recall the basic notions of holomorphic curves in quaternionic projective space $\mathbb{H} \mathbb{P}^{n}$ and explain briefly the Kodaira correspondence [FLPP01] which relates holomorphic curves in $\mathbb{H} \mathbb{P}^{n}$ to quaternionic holomorphic line bundles with $(n+1)$-dimensional subspaces of holomorphic sections, the so called linear systems.

A map $f: M \rightarrow \mathbb{H}^{n}$ of a Riemann surface $M$ into quaternionic projective space $\mathbb{H} \mathbb{P}^{n}$ is the same as the line subbundle $L \subset V=\underline{\mathbb{H}}^{n+1}$ of the trivial $\mathbb{H}^{n+1}$-bundle whose fiber over $p$ is given by $L_{p}=f(p)$, i.e., $L=f^{*} \mathcal{T}$ is the pull-back of the tautological line bundle $\mathcal{T}$ over $\mathbb{H P}^{n}$. A smooth map $f: M \rightarrow \mathbb{H}^{n}$ is called a holomorphic curve if the 
line bundle $L$ has a complex structure $J \in \Gamma(\operatorname{End}(L)), J^{2}=-1$, such that $* \delta=\delta J$. Here $\delta=\left.\pi_{L} \nabla\right|_{L} \in \Omega^{1}(\operatorname{Hom}(L, V / L))$ denotes the derivative of $f$ with $\pi_{L}: V \rightarrow V / L$ the canonical projection and $\nabla$ the trivial connection on $V$. In the case $n=1$ a holomorphic curve is a conformal map $f: M \rightarrow S^{4}$ into the 4-sphere.

A quaternionic holomorphic structure on a complex quaternionic line bundle $(L, J)$ is a quaternionic linear map $D: \Gamma(L) \rightarrow \Gamma(\bar{K} L)$ which satisfies the Leibniz rule

$$
D(\psi \lambda)=(D \psi) \lambda+(\psi d \lambda)^{\prime \prime},
$$

where $\psi \in \Gamma(L)$ is a section of $L$ and $\lambda: M \rightarrow \mathbb{H}$ is a quaternionic valued function. We denote by $\omega^{\prime} \in \Gamma(K W)$ and $\omega^{\prime \prime} \in \Gamma(\bar{K} W)$ the $(1,0)$ and $(0,1)$-part of a 1 -form $\omega \in \Omega^{1}(W)$ with values in a complex vector bundle $W$. Let

$$
H^{0}(L)=H^{0}(L, D)=\{\psi \in \Gamma(L) \mid D \psi=0\}
$$

be the space of holomorphic sections of $L$ with respect to $D$. An example of a holomorphic structure is given by the $(0,1)$-part $\left(\nabla^{L}\right)^{\prime \prime}$ of a quaternionic connection $\nabla^{L}$ on $L$.

A holomorphic curve $f: M \rightarrow \mathbb{H}^{n}$ induces by [FLPP01] a unique holomorphic structure $D$ on the dual bundle $L^{-1}$ of $L=f^{*} \mathcal{T}$ such that linear forms $\alpha \in V^{*}$ on $V$ restricted to $L$ give holomorphic sections

$$
\left.\alpha\right|_{L} \in H^{0}\left(L^{-1}\right)
$$

Thus $H=V^{*} \subset H^{0}\left(L^{-1}\right)$ is a $(n+1)$-dimensional linear system. By transversality $H$ is basepoint free, in other words, there exists a nowhere vanishing holomorphic section of the holomorphic line bundle $L^{-1}$ in $H$. Conversely, a holomorphic line bundle $L^{-1}$ and a basepoint free $(n+1)$-dimensional linear system $H \subset H^{0}\left(L^{-1}\right)$ give a holomorphic curve $f: M \rightarrow \mathbb{H}^{n}=\mathbb{P}\left(H^{*}\right)$ by

$$
\mathrm{ev}^{*}(L) \subset V=(M \times H)^{*},
$$

which is called the Kodaira embedding of $L$ with respect to the linear system $H$. Here the bundle map

$$
\mathrm{ev}: M \times H \rightarrow L^{-1},(p, \psi) \mapsto \psi_{p}
$$

evaluates the holomorphic section $\psi$ at the point $p$.

In particular, every holomorphic curve $f: M \rightarrow \mathbb{H} \mathbb{P}^{n}$ gives rise to a family of conformal maps $f^{\sharp}: M \rightarrow S^{4}$ : choosing a 2-dimensional linear system $H^{\sharp} \subset H$ yields, via the Kodaira embedding of $L \subset V^{\sharp}=\left(H^{\sharp}\right)^{*}$, a conformal map $f^{\sharp}: M \rightarrow S^{4}$.

In what follows, we frequently will make use of the bundle $K L$ of $L$-valued $(1,0)$ forms. By [PP98] there is a unique holomorphic structure $D^{K L}$ on $K L$ compatible with the pairing

$$
L^{-1} \times K L \rightarrow \Lambda^{2} T M^{*} \otimes \mathbb{H}:
$$

given $\psi \in \Gamma\left(L^{-1}\right)$ and $\omega \in \Gamma(K L)$ the holomorphic structure is determined by the Leibniz rule

$$
d\langle\psi, \omega\rangle=\langle D \psi \wedge \omega\rangle+\left\langle\psi, D^{K L} \omega\right\rangle .
$$

Two descriptions of $D^{K L}$ will be useful for our purposes: first, since $L \subset V$ is a subbundle, we can take exterior derivatives of $\omega \in \Gamma(K L)$ with respect to the trivial 
connection $\nabla$ on $V$. But $\pi d^{\nabla} \omega=\delta \wedge \omega=0$ by type considerations which implies that $d^{\nabla} \omega \in \Omega^{2}(L)$ is again $L$-valued. One immediately checks (1) for $d^{\nabla}$ and thus

$$
D^{K L}=d^{\nabla} \text {. }
$$

Second, if $\nabla^{L^{-1}}$ is any connection on $L^{-1}$ adapted to the holomorphic structure $D$ on $L^{-1}$, i.e., $D=\left(\nabla^{L^{-1}}\right)^{\prime \prime}$, then the exterior derivative of $\omega \in \Gamma(K L)$ with respect to the dual connection $\nabla^{L}$ on $L$ also gives

$$
D^{K L}=d^{\nabla^{L}} .
$$

This follows again from the fact that $d^{\nabla^{L}}$ satisfies (1).

To construct a Bäcklund transform of a holomorphic curve $f: M \rightarrow \mathbb{H} \mathbb{P}^{n}$, we first fix a hyperplane at infinity: the basepoint free linear system $H$ has a nowhere vanishing holomorphic section and thus we can choose $\alpha \in V^{*}$ so that $V=L \oplus \operatorname{ker} \alpha$. In this splitting we decompose the trivial connection $\nabla$ on $V$ as

$$
\left.\nabla\right|_{L}=\nabla^{L}+\delta
$$

Here $\delta$ is the derivative of $f$ expressed via the identification $V / L=\operatorname{ker} \alpha$ and the induced connection $\nabla^{L}$ on $L$ is flat since $\operatorname{ker} \alpha \subset V$ is constant. We now equip the line bundle $L$ with the holomorphic structure

$$
D=\left(\nabla^{L}\right)^{\prime \prime}
$$

coming from the connection $\nabla^{L}$ on $L$. Note that $L$ always has the canonical nowhere vanishing holomorphic section $\psi \in H^{0}(L)$ for which $\langle\alpha, \psi\rangle=1$ : since $\nabla^{L} \psi=0$ the section $\psi$ also has $D \psi=0$ and thus is holomorphic. In particular, the complete linear system $H^{0}(L)$ is basepoint free.

Definition 2.1. Let $f: M \rightarrow \mathbb{H} \mathbb{P}^{n}$ be a holomorphic curve and $\alpha \in V^{*}$ such that $V=L \oplus \operatorname{ker} \alpha$. If $\tilde{H} \subset H^{0}(L)$ is a basepoint free linear system of dimension at least 2 , the holomorphic curve

$$
B_{\alpha, \tilde{H}}(f)=\tilde{f}: M \rightarrow \mathbb{P}\left(\tilde{H}^{*}\right),
$$

obtained via the Kodaira embedding of $L^{-1}$ in $\tilde{H}^{*}$, is a Bäcklund transform of $f$ (with parameters $\alpha$ and $\tilde{H})$. If $\tilde{H}=H^{0}(L)$ is the complete linear system, we call $\tilde{f}=B_{\alpha}(f)$ the Bäcklund transform of $f$.

To estimate the dimension $h^{0}(L)$ of the space of holomorphic sections of $L$, in the case when $M$ is compact of genus $g$, we use the Riemann-Roch Theorem [PP98] for quaternionic holomorphic line bundles:

$$
h^{0}(L)-h^{0}\left(K L^{-1}\right)=\operatorname{deg} L-g+1 .
$$

The degree of the complex quaternionic line bundle $L=E \oplus E$ is defined as the degree of the underlying complex line bundle $E$, in other words, as the degree of the $+i$-eigenspace $E$ of $J$ on $L$, [PP98]. From (3) we know that the holomorphic structure on $K L^{-1}$ is given by the exterior derivative $d^{\nabla^{-1}}$ with respect to the dual connection $\nabla^{L^{-1}}$ on $L^{-1}$. Since $\left.\alpha\right|_{L} \in H^{0}\left(L^{-1}\right)$ is parallel with respect to $\nabla^{L^{-1}}$ the linear map

$$
H^{0}\left(L^{-1}\right) \rightarrow H^{0}\left(K L^{-1}\right): \beta \mapsto \nabla^{L^{-1}} \beta
$$


has kernel spanned by $\left.\alpha\right|_{L}$ and we obtain

$$
h^{0}\left(K L^{-1}\right)=h^{0}\left(L^{-1}\right)-1 \geq n .
$$

Applying the Riemann-Roch relation (6) we get:

THEOREM 2.2. Let $M$ be a compact Riemann surface of genus $g$ and let $f: M \rightarrow \mathbb{H}^{n}$ be a holomorphic curve. For every choice of $\alpha \in V^{*}$ with $\left.\alpha\right|_{L} \in H^{0}\left(L^{-1}\right)$ nowhere vanishing, there is an induced flat connection $\nabla^{L}$ on $L$ given by the splitting $V=L \oplus \operatorname{ker} \alpha$.

If $L$ is equipped with the holomorphic structure $D=\left(\nabla^{L}\right)^{\prime \prime}$, then the dimension of the space of holomorphic sections of $L$ is given by

$$
h^{0}(L)=h^{0}\left(L^{-1}\right)+\operatorname{deg} L-g .
$$

In particular, if the degree of the line bundle $L=f^{*} \mathcal{T}$ satisfies

$$
\operatorname{deg} L \geq 1+g-n
$$

then the complete linear system $H^{0}(L)$ is at least 2-dimensional and there exists a Bäcklund transform of $f$.

In order to see how the Willmore energy behaves under Bäcklund transformation, we first recall the Willmore energy

$$
W(f)=2 \int_{M}\langle Q \wedge * Q\rangle
$$

of a holomorphic curve $f: M \rightarrow \mathbb{H}^{n}$ of a compact Riemann surface into quaternionic projective space $\mathbb{H P}^{n}[\mathrm{FLPP} 01]$. Here $\langle B\rangle=\operatorname{tr}_{\mathbb{R}} B$ is the real trace of an endomorphism $B \in \operatorname{End}(V)$ and $Q \in \Gamma\left(\bar{K} \operatorname{End}_{-}\left(L^{-1}\right)\right)$ is given by the splitting of the holomorphic structure $D=\bar{\partial}+Q$ on $L^{-1}$ into its $J$-commuting and anticommuting parts. Furthermore, we denote by $\mathrm{Hom}_{ \pm}$the complex linear respectively antilinear homomorphisms. In case $f: M \rightarrow S^{4}=\mathbb{H P}^{1}$ is a conformal immersion it is shown in $\left[\mathrm{BFL}^{+} 02\right]$ that the energy (7) coincides with the classical Willmore energy

$$
W(f)=\int_{M}|H|^{2}-K-K^{\perp},
$$

where $H$ is the mean curvature vector, $K$ the Gaussian curvature and $K^{\perp}$ the curvature of the normal bundle of $f$.

TheOREM 2.3. Let $f: M \rightarrow \mathbb{H}^{n}$ be a holomorphic curve, $M$ a compact Riemann surface, and let $B_{\alpha, \tilde{H}}(f): M \rightarrow \mathbb{H}^{k}$ be a Bäcklund transform of $f$ with respect to an $\alpha \in V^{*}$ and $\tilde{H} \subset H^{0}(L)$. Then the Willmore energy of $B_{\alpha, \tilde{H}}(f)$ is given by

$$
W\left(B_{\alpha, \tilde{H}}(f)\right)=W(f)-4 \pi \operatorname{deg} L .
$$

REMARK 2.4. This formula seems to suggest that the Bäcklund transform of a holomorphic curve $f: M \rightarrow \mathbb{H} \mathbb{P}^{n}$ which is Willmore [LP03] is again Willmore, an issue which we will return to in Section 5.

Proof. The flat connection $\nabla^{L}=\hat{\nabla}+Q+A$ on $L$ splits into a complex connection $\hat{\nabla}$ and the $J$-anticommuting endomorphisms $A \in \Gamma\left(K\right.$ End $\left._{-}(L)\right)$ and $Q \in \Gamma\left(\bar{K}\right.$ End $\left.\left.{ }_{-} L\right)\right)$. 
Therefore, the $J$-anticommuting part of the holomorphic structure on $L$ is given by $\left(\nabla^{L}\right)_{-}^{\prime \prime}=Q$ and the Willmore energy of the Bäcklund transform $B_{\alpha, \tilde{H}}(f)$ is given by

$$
W\left(B_{\alpha, \tilde{H}}(f)\right)=2 \int_{M}\langle Q \wedge * Q\rangle .
$$

Since $\nabla^{L}$ is flat, we have

$$
0=R^{\nabla^{L}}=R^{\hat{\nabla}}+d^{\hat{\nabla}}(A+Q)+\frac{1}{2}[A+Q \wedge A+Q] .
$$

Taking the $J$-commuting part of this equation gives

$$
0=R^{\hat{\nabla}}+A \wedge A+Q \wedge Q
$$

which shows that

$$
W\left(B_{\alpha}(f)\right)=2 \int_{M}\langle A \wedge * A\rangle-\left\langle J R^{\hat{\nabla}}\right\rangle .
$$

Since $\hat{\nabla}$ is a complex connection on $L$, we have $2 \pi \operatorname{deg} L=\int_{M}\left\langle J R^{\hat{\nabla}}\right\rangle$ and it remains to verify that

$$
W(f)=2 \int_{M}\langle A \wedge * A\rangle .
$$

The section $\left.\alpha\right|_{L} \in H^{0}\left(L^{-1}\right)$ is a parallel section of the dual connection $\nabla^{L^{-1}}$ of $\nabla^{L}$. Therefore, $\nabla^{L^{-1}}$ induces the given holomorphic structure $D$ on $L^{-1}$ and

$$
D=\left(\nabla^{L^{-1}}\right)^{\prime \prime}=\bar{\partial}-A^{*},
$$

which implies that $W(f)=2 \int_{M}\langle A \wedge * A\rangle$.

REMARK 2.5. If we apply a Bäcklund transformation to $B_{\alpha}(f)$ with respect to the holomorphic section $\psi \in H^{0}(L)$ with $\langle\alpha, \psi\rangle=1$, then the holomorphic structure on $L^{-1}$ induced by $\psi$ satisfies $D \alpha=0$. Hence $D$ is the given holomorphic structure on $L^{-1}$. This shows that $B_{\psi, H}\left(B_{\alpha}(f)\right)=f$ where $H \subset H^{0}\left(L^{-1}\right)$ is the linear system of $f$. On the other hand, if we choose arbitrary nowhere vanishing $\varphi \in H^{0}(L)$ the induced holomorphic structure on $L^{-1}$ will differ from $D$, and we get a transformation on holomorphic curves preserving the Willmore energy by Theorem 2.3.

3. Construction of Bäcklund transforms from abelian integrals. The construction of a Bäcklund transform of a holomorphic curve $f: M \rightarrow \mathbb{H} \mathbb{P}^{n}$ involves two choices: first a hyperplane ker $\alpha \subset V$ at $\infty$ not intersecting $f$ given by $\alpha \in V^{*}$ so that $V=L \oplus \operatorname{ker} \alpha$ and, secondly, a choice of a basepoint free linear system $\tilde{H} \subset H^{0}(L)$ of dimension at least 2. Then the Kodaira embedding $\tilde{f}: M \rightarrow \mathbb{P}\left(\tilde{H}^{*}\right)$ of $L$ with respect to the linear system $\tilde{H}$ is the Bäcklund transform $B_{\alpha, \tilde{H}}(f)$ of $f$.

We now will explain how one can use abelian integrals to construct linear systems $\tilde{H} \subset H^{0}(L)$. The holomorphic structure on $L$ is induced by the splitting $V=L \oplus \operatorname{ker} \alpha$ and is given (4), (5) by the $(0,1)$-part of the flat connection $\nabla^{L}$ on $L$. On the other hand, the bundle $K L$ has a canonical holomorphic structure entirely determined by the holomorphic curve $f: M \rightarrow \mathbb{H} \mathbb{P}^{n}$ expressed by (3). Therefore, the integrals of holomorphic sections $\omega \in H^{0}(K L)$ give holomorphic sections $\varphi \in H^{0}(L)$-at least on the universal 
cover pr $: \tilde{M} \rightarrow M$. Since the section $\psi \in H^{0}(L)$ with $\langle\alpha, \psi\rangle=1$ has no zeros any linear system $\tilde{H} \subset H^{0}\left(\mathrm{pr}^{*} L\right)$ containing $\psi$ is basepoint free.

Given a linear system $H_{K L} \subset H^{0}(K L)$ the linear system $\tilde{H} \subset H^{0}\left(\mathrm{pr}^{*} L\right)$ obtained by integrating sections in $H_{K L}$ and including the section $\psi$ with $\langle\alpha, \psi\rangle=1$ is called the linear system obtained by integration of $H_{K L}$. Since we have included $\psi \in H^{0}(L)$, which appears as the constant of integration, in the linear system $\tilde{H}$ this procedure is well-defined. Moreover, because $\psi \in \tilde{H}$ has no zeros, the linear system $\tilde{H}$ is basepoint free. To calculate the dimension of $\tilde{H}$ in case $M$ is compact and $H_{K L}=H^{0}(K L)$ is the complete linear system, we use the Riemann-Roch theorem (6) applied to $L^{-1}$ :

$$
h^{0}(K L)=h^{0}\left(L^{-1}\right)+\operatorname{deg} L+g-1
$$

and therefore

$$
\operatorname{dim} \tilde{H}=1+h^{0}(K L)=h^{0}\left(L^{-1}\right)+\operatorname{deg} L+g .
$$

Lemma 3.1. Let $f: M \rightarrow \mathbb{H P}^{n}$ be a holomorphic curve. Then, for any choice of hyperplane $\alpha \in V^{*}$ not intersecting $f$, the linear system $\tilde{H} \subset H^{0}\left(\mathrm{pr}^{*} L\right)$ obtained by integration of $H^{0}(K L)$ is basepoint free and has dimension

$$
\operatorname{dim} \tilde{H}=h^{0}\left(L^{-1}\right)+\operatorname{deg} L+g .
$$

Assuming that $m+1=\operatorname{dim} \tilde{H} \geq 2$, we obtain a Bäcklund transform $\tilde{f}=B_{\alpha, \tilde{H}}(f): \tilde{M} \rightarrow$ $\mathbb{H}^{m}$ on the universal cover $\tilde{M}$ of $M$.

If we only are concerned about local surface theory then spaces of holomorphic sections are infinite dimensional, abelian integrals have no periods, and we always obtain Bäcklund transforms by integrating sections in $H^{0}(K L)$.

In the case of compact surfaces, genus 0 is exceptional since there are no periods to close. Moreover, for a holomorphic sphere $f: S^{2} \rightarrow \mathbb{H} \mathbb{P}^{n}$ of

$$
\operatorname{deg} L \geq-n+1
$$

the previous Lemma implies that $m+1=\operatorname{dim} \tilde{H} \geq 2$ since $h^{0}\left(L^{-1}\right) \geq n+1$. Thus we always have Bäcklund transforms $\tilde{f}: S^{2} \rightarrow \mathbb{H} \mathbb{P}^{m}$. In [Pet04], [BP] the Bäcklund transformation is used to construct soliton spheres.

For higher genus surfaces $f: M \rightarrow \mathbb{H P}^{n}$, we have to close the periods of the Bäcklund transform. If $H_{K L} \subset H^{0}(K L)$ is a linear system of $\operatorname{dim} H_{K L} \leq n+1$, we choose a linear map $\Omega: V \rightarrow H^{0}(K L)$ whose image is $H_{K L}$. To obtain a closed Bäcklund transform it suffices to find $\alpha \in V^{*}$ with $V=L \oplus \operatorname{ker} \alpha$ such that the periods

$$
\left\langle\alpha, \int_{\gamma} \Omega\right\rangle=0
$$

vanish for all $\gamma \in \pi_{1}(M)$.

In the case when $M=T^{2}$ is a torus it is possible to find $\alpha \in V^{*}$ and linear systems $H_{K L} \subset H^{0}(K L)$ for which (9) holds: since $\pi_{1}\left(T^{2}\right)=\mathbb{Z} \gamma_{1} \oplus \mathbb{Z} \gamma_{2}$ we have two period maps $P_{i}=\int_{\gamma_{i}} \Omega \in \operatorname{End}(V)$. If one of the $P_{i}$, say $P_{1}$, has kernel we choose a linear subspace $V_{1} \subset \operatorname{ker} P_{1}$ of $\operatorname{dim} V_{1}<\operatorname{dim} V$ and $\alpha \in V^{*}$ with $\left\langle\alpha, P_{2}\left(V_{1}\right)\right\rangle=0$. Then the linear system $H_{1}=\Omega\left(V_{1}\right) \subset H_{0}(K L)$ has no periods. If $P_{1}$ is invertible, then $P_{1}^{-1} P_{2}$ has a fixed space 
of $\operatorname{dimension} 1 \leq \operatorname{dim} V_{2}<\operatorname{dim} V$. Then $P_{1}\left(V_{2}\right)=P_{2}\left(V_{2}\right)$ and we may choose $\alpha \in V^{*}$ with $\left\langle\alpha, P_{1}\left(V_{2}\right)\right\rangle=0$. Again, the linear system $H_{2}=\Omega\left(V_{2}\right) \subset H^{0}(K L)$ has no periods.

To integrate the linear system $H_{i} \subset H^{0}(K L)$, we need $\alpha \in V^{*}$ to satisfy $V=L \oplus \operatorname{ker} \alpha$. Since we cannot choose $\alpha \in V^{*}$ freely this may not be the case for our $\alpha \in V^{*}$ resulting in a discrete set of zeros of $\left.\alpha\right|_{L} \in H^{0}\left(L^{-1}\right)$. In this case the splitting $V=L \oplus \operatorname{ker} \alpha$ is not defined everywhere. Thus we do not get the flat connection $\nabla^{L}$ on $L$ needed to define the holomorphic structure on $L$ and to integrate sections in $H^{0}(K L)$ to a linear system $\tilde{H} \subset H^{0}(L)$. To remedy this situation, we describe an alternative way to obtain the Bäcklund transformation given by abelian integrals of a linear system $H_{K L} \subset H^{0}(K L)$. By (2) an $L$-valued $(1,0)$-form $\omega \in H^{0}(K L)$ is holomorphic if it is closed as a $V$-valued form, i.e.,

$$
d^{\nabla} \omega=0 .
$$

Thus, given a linear system $H_{K L} \subset H^{0}(K L)$ and any $\alpha \in V^{*}$, we can integrate $\left\langle\alpha, \omega_{i}\right\rangle=$ $d \bar{g}_{i}$ with $g_{i}: \tilde{M} \rightarrow \mathbb{H}$. The smooth map

$$
g=\left[g_{1}: \ldots: g_{k}: 1\right]: \tilde{M} \rightarrow \mathbb{H}^{k}
$$

is well-defined up to projective equivalence (resulting from different choices of basis $\omega_{i} \in$ $H^{0}(K L)$ and constants of integration) and is called a generalized Bäcklund transform of $f$. It is now easy to see that $g$ coincides with the Bäcklund transform $B_{\alpha, \tilde{H}}(f)$ in case $\alpha \in V^{*}$ has no zeros on $L$. In the case when $\alpha \in V^{*}$ has zeros on $L$ the generalized Bäcklund transform is a holomorphic curve away from those zeros: the complex structure on the bundle $g^{*} \mathcal{T}$ is given by

$$
J\left(\begin{array}{c}
g_{1} \\
\vdots \\
g_{n} \\
1
\end{array}\right)=\left(\begin{array}{c}
g_{1} \\
\vdots \\
g_{n} \\
1
\end{array}\right) N
$$

where $N$ is defined by $\left.J \alpha\right|_{L}=\left.\alpha\right|_{L} N$ away from the zeros of $\alpha$.

4. Geometric interpretation of Bäcklund transforms. In this section we give a geometric interpretation for the Bäcklund transformation using the enveloping construction [LP03]. A holomorphic curve $f: M \rightarrow \mathbb{H} \mathbb{P}^{n}$ has the osculating flag $L=f^{*} \mathcal{T} \subset V_{1} \subset$ $\ldots \subset V_{n-1} \subset V=\underline{\mathbb{H}}^{n+1}$ given by

$$
\nabla\left(\Gamma\left(V_{k}\right)\right) \subset \Omega^{1}\left(V_{k+1}\right),
$$

which exists away from the discrete set of Weierstrass points into which the flag extends continuously [FLPP01]. The construction of an envelope of the holomorphic curve $f$ requires that the osculating flag exists smoothly on $M$. In view of applications to Willmore surfaces, we restrict to the even smaller class of Frenet curves $f: M \rightarrow \mathbb{H}^{n}$. Recall [LP03] that a holomorphic curve $f: M \rightarrow \mathbb{H}^{n}$ is a Frenet curve if there is a smooth flag $L \subset V_{1} \subset \ldots \subset V_{n-1} \subset V=\underline{\mathbb{H}}^{n+1}$ and a complex structure $S \in \Gamma(\operatorname{End}(V))$ such that

$$
* \delta_{k}=S \delta_{k}=\delta_{k} S
$$


and

$$
\left.(\nabla S)^{\prime \prime}\right|_{V_{n-1}}=0, \quad \text { or, equivalently, } \quad(\nabla S)^{\prime} V \subset L .
$$

Here $\delta_{k}=\left.\pi_{V_{k}} \nabla\right|_{V_{k}} \in \Gamma\left(K \operatorname{Hom}_{+}\left(V_{k}, V_{k+1} / V_{k}\right)\right)$ denote the derivatives of $V_{k}$ with $\pi_{V_{k}}$ : $V \rightarrow V / V_{k}$ the canonical projections and $\nabla$ the trivial connection on $V$. The unique complex structure $S \in \Gamma(\operatorname{End}(V))$ given by (11) and (12) is called the canonical complex structure [FLPP01] of the Frenet curve $f$. The dual curve $f^{\perp}$ of a Frenet curve is defined by the line bundle $V_{n-1}^{\perp} \subset V^{*}$. This is again a Frenet curve with derivatives $\delta_{k}^{\perp}=-\delta_{n-k-1}^{*}$ and canonical complex structure $S^{*}$.

If $f: M \rightarrow S^{4}=\mathbb{H}^{1}$ is a conformal immersion, the canonical complex structure $S \in \Gamma(\operatorname{End}(V))$ can be seen as the mean curvature sphere congruence of $f$ : at each point $p \in M$, we have a sphere $S^{\prime}(p)$ given by the fixed lines $S^{\prime}(p)=\left\{l \in \mathbb{H} \mathbb{P}^{1} \mid S(p) l=l\right\}$ of $S(p)$. The conditions (11) and (12) imply [BFL $\left.{ }^{+} 02\right]$ that the sphere $S^{\prime}(p)$ goes through $f(p)$, touches $f$ at $f(p)$ and has the same mean curvature vector as $f$ at $f(p)$.

Given a Frenet curve $\hat{f}: M \rightarrow \mathbb{H}^{n+1}$ we get [LP03] a holomorphic curve $f: M \rightarrow$ $\mathbb{H} \mathbb{P}^{n}$, the tangent curve of $\hat{f}$, by intersecting the first flag space $\hat{V}_{1}$ of the Frenet flag of $\hat{f}$ with a $\mathbb{H} \mathbb{P}^{n} \subset \mathbb{H} \mathbb{P}^{n+1}$. Conversely, $\hat{f}: M \rightarrow \mathbb{H}^{n+1}$ is called an envelope of $f: M \rightarrow \mathbb{H}^{n}$ if $f$ is a tangent curve of $\hat{f}$.

For a Frenet curve $f: M \rightarrow \mathbb{H}^{n}$ with corresponding line bundle $L \subset V$ any choice of a nowhere vanishing holomorphic section $\omega \in H^{0}(K L)$ gives an envelope: if $\varphi \in \Gamma(V)$ satisfies $\nabla \varphi=-\omega$ then $\hat{\psi}=\varphi \oplus 1$ is a nowhere vanishing section of the trivial $\mathbb{H}^{n+1}$ bundle $\hat{V}=\operatorname{pr}^{*}(V) \oplus \underline{\mathbb{H}}$ over the universal cover $\tilde{M}$ of $M$, and defines the quaternionic line bundle $\hat{L}=\hat{\psi} \mathbb{H} \subset \overline{\hat{V}}$. For $\hat{\alpha} \in \hat{V}^{*}$ nowhere vanishing and ker $\hat{\alpha}=V$ we see that

$$
L=\operatorname{ker} \hat{\alpha} \cap(\hat{L} \oplus \operatorname{im} \hat{\delta})=\operatorname{ker} \hat{\alpha} \cap V_{1} \subset V,
$$

since $\hat{\delta} \hat{\psi}=-\omega \in H^{0}(K L)$. Moreover, $\omega$ defines $N \in \operatorname{Im} \mathbb{H}$ by $* \omega=\omega N$ and gives a complex structure $J$ on $\hat{L}$ by $J \hat{\psi}=\hat{\psi} N$. In particular, $\hat{L}$ is a holomorphic curve $\hat{f}: \tilde{M} \rightarrow \mathbb{H} \mathbb{P}^{n+1}$, and due to (13) an envelope of $f$. It can be shown that $\hat{f}$ is in fact a Frenet curve [LP03].

THEOREM 4.1. Every Bäcklund transform $\tilde{f}$ of a Frenet curve $f: M \rightarrow \mathbb{H}^{n}$ which is given by integration of a linear system $H_{K L}=\operatorname{span}\{\omega\}$ with nowhere vanishing $\omega \in$ $H^{0}(K L)$ is the dual curve of a projection of an envelope $\hat{f}$ of $f$. The envelope $\hat{f}: \tilde{M} \rightarrow$ $\mathbb{H} \mathbb{P}^{n+1}$ is defined by $\hat{L}=\hat{\psi} \mathbb{H} \subset \underline{\mathbb{H}}^{n+2}$ with $\nabla \hat{\psi}=-\omega$.

Proof. Let $\hat{\alpha} \in \hat{V}^{*}$ with $\left.\hat{\alpha}\right|_{\hat{L}}$ never zero and ker $\hat{\alpha}=V$. Since

$$
d\langle\hat{\alpha}, \hat{\psi}\rangle=\langle\hat{\alpha}, \nabla \hat{\psi}\rangle=-\langle\hat{\alpha}, \omega\rangle=0
$$

we can assume, by scaling $\hat{\alpha}$ if necessary, that $\langle\hat{\alpha}, \hat{\psi}\rangle=1$. Choose $\alpha_{1} \in \hat{V}^{*}$ such that $\alpha=\left.\alpha_{1}\right|_{V}$ is nowhere vanishing on $L$, and denote by $H^{\sharp}$ the linear system spanned by $\hat{\alpha}$ and $\alpha_{1}$. The linear system $H^{\sharp}$ is basepoint free since $\hat{\alpha}$ has no zeros. In particular, the Kodaira embedding of $\hat{L}$ in $\left(H^{\sharp}\right)^{*}$ is a holomorphic curve $f^{\sharp}: \tilde{M} \rightarrow \mathbb{H} \mathbb{P}^{1}=\mathbb{P}\left(\left(H^{\sharp}\right)^{*}\right)$. Note that

$$
\left.\alpha_{1}\right|_{\hat{L}}=-\left.\hat{\alpha}\right|_{\hat{L}} g,
$$

where $g: \tilde{M} \rightarrow \mathbb{H}$ satisfies $d \bar{g}=-d\left\langle\alpha_{1}, \hat{\psi}\right\rangle=-\left\langle\alpha_{1}, \nabla \hat{\psi}\right\rangle=\langle\alpha, \omega\rangle$. 
We now show that $f^{\sharp}$ is the dual curve of the Bäcklund transform $\tilde{f}$. Recall that the dual curve $\tilde{f}^{\perp}$ of $\tilde{f}$ is given by the Kodaira embedding of $\left(L^{-1}\right)^{\perp}$ in $\tilde{H}^{*}$, where the linear system $\tilde{H}$ obtained by integration of $H_{K L}$ is given by $\tilde{H}=\operatorname{span}\{\psi \bar{g}, \psi\}$. Therefore, the dual basis $\{\tilde{\alpha}, \tilde{\beta}\} \subset \tilde{H}^{*}$ of $\{\psi \bar{g}, \psi\}$ satisfies

$$
\left.\tilde{\beta}\right|_{\left(L^{-1}\right)^{\perp}}=-\left.\tilde{\alpha}\right|_{\left(L^{-1}\right)^{\perp}} g .
$$

In particular, the quaternionic linear map

$$
\rho: \hat{L}^{-1} \rightarrow\left(\left(L^{-1}\right)^{\perp}\right)^{-1}
$$

defined by $\rho(\hat{\alpha})=\tilde{\alpha}$ is a holomorphic bundle map which maps the linear system $H^{\sharp}$ to the linear system $\tilde{H}^{*}$ which shows that the dual curve $\tilde{f}^{\perp}: \tilde{M} \rightarrow \mathbb{P}\left(\tilde{H}^{*}\right)$ is given by $f^{\sharp}: \tilde{M} \rightarrow \mathbb{P}\left(\left(H^{\sharp}\right)^{*}\right)$.

REMARK 4.2. The assumption of the theorem can be relaxed: as we have seen, an envelope of a holomorphic curve exists if the osculating flag exists smoothly on $M$. Moreover, if $f: M \rightarrow \mathbb{H} \mathbb{P}^{n}$ is Willmore then there are canonical choices of $\omega \in H^{0}(K L)$ for which it is still possible to define the canonical complex structure of the envelope even though these $\omega$ might have zeros, [LP03].

5. Bäcklund transforms of Willmore surfaces. As an application we discuss Bäcklund transforms of Willmore surfaces. Recall that a Frenet curve $f: M \rightarrow \mathbb{H}^{n}$ from a compact Riemann surface $M$ to quaternionic projective space $\mathbb{H} \mathbb{P}^{n}$ is called Willmore if it is a critical point of the Willmore energy (7) under compactly supported variations by Frenet curves where the conformal structure of $M$ may change under the variation [LP03]. This definition coincides with the classical definition of Willmore surfaces for conformal immersions $f: M \rightarrow S^{4}$ and includes the case of those branched conformal immersions for which the mean curvature sphere extends smoothly into the branch points.

It is a classical fact that the mean curvature sphere congruence of a Willmore surface is harmonic. This fact also holds true in the general case of Willmore surfaces in quaternionic projective space [LP03]: a Frenet curve $f: M \rightarrow \mathbb{H} \mathbb{P}^{n}$ is Willmore if and only if the canonical complex structure of $f$ is harmonic. If $S \in \Gamma(\operatorname{End}(V))$ is a complex structure, we split $\nabla S$ into its $(0,1)$ and $(1,0)$-parts, i.e.,

$$
\nabla S=2(* Q-* A),
$$

where $Q \in \Gamma\left(\bar{K}\right.$ End $\left._{-}(V)\right)$ and $A \in \Gamma\left(K\right.$ End $\left._{-}(V)\right)$. With this notation the harmonicity of the canonical complex structure $S$ of a Frenet curve $f: M \rightarrow \mathbb{H} \mathbb{P}^{n}$ reads as

$$
d^{\nabla} * A=0 \quad \text { or, equivalently, } \quad d^{\nabla} * Q=0 .
$$

Holomorphic curves with $A \equiv 0$ are [FLPP01] exactly the twistor projections of holomorphic curves in $\mathbb{C P}^{2 n+1}$. It turns out that envelopes and tangents, and thus also Bäcklund transforms, of Willmore curves are not necessarily Willmore. Rather they become what is known to be constrained Willmore surfaces:

Definition 5.1. A Frenet curve $f: M \rightarrow \mathbb{H}^{n}$ with canonical complex structure $S$ is called constrained Willmore if there exists $\eta \in \Gamma\left(K \mathcal{R}_{+}\right)$with 


$$
d^{\nabla}(* A+\eta)=0
$$

Here $\mathcal{R}=\operatorname{Hom}\left(V / V_{n-1}, L\right)$ and $L \subset V_{1} \subset \ldots \subset V_{n-1} \subset V$ is the Frenet flag of $f$.

It can be shown by methods similar to the ones in [LP03] that every constrained Willmore curve is a critical point of the Willmore functional under variations by Frenet curves which preserve the conformal structure of $M$.

In the case of constrained Willmore surfaces with $A \not \equiv 0$ there is a canonical linear map $\Omega: V \rightarrow H^{0}(K L)$, namely

$$
\Omega=* A+\eta,
$$

which is non-trivial since $A$ and $\eta$ are of different \pm -type. Therefore, we can apply Lemma 3.1 to construct Bäcklund transforms by abelian integrals:

Corollary 5.2. Let $f: M \rightarrow \mathbb{H}^{n}$ be constrained Willmore so that

$$
d^{\nabla}(* A+\eta)=0 \quad \text { and } \quad A \not \equiv 0 .
$$

Then for any choice of $\alpha \in V^{*}$ with $V=L \oplus \operatorname{ker} \alpha$ the linear system $H_{K L}=\{(* A+\eta) b \mid$ $b \in V\} \subset H^{0}(K L)$ has dimension $m=\operatorname{dim} H_{K L} \geq 1$ and there exists a Bäcklund transform

$$
\tilde{f}: \tilde{M} \rightarrow \mathbb{H} \mathbb{P}^{m}
$$

on the universal cover $\tilde{M}$ of $M$ given by integration of $H_{K L}$.

Moreover, in case of a torus $M=T^{2}$ there exists a (generalized) closed Bäcklund transform.
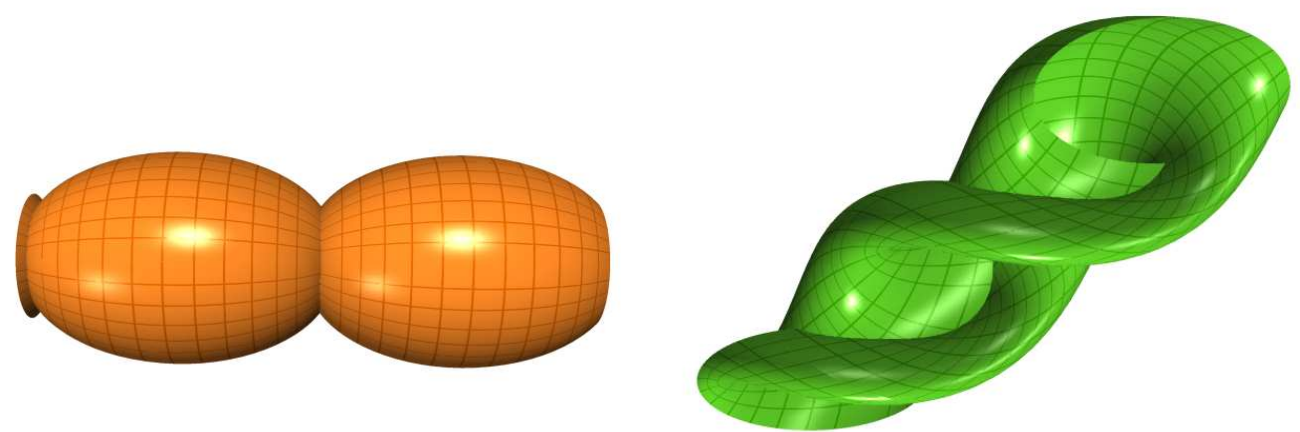

Fig. 1. Willmore cylinders in $\mathbb{R}^{4}$ obtained by Bäcklund transformation of the Clifford torus, [Hel02]

We now show, at least in the case of a conformal immersion $f: M \rightarrow S^{4}$, that the Bäcklund transforms with respect to the linear map $\Omega=* A+\eta$ preserve the constrained Willmore and also the Willmore property. The latter has already been shown in $\left[\mathrm{BFL}^{+} 02\right]$.

TheOREM 5.3. Let $f: M \rightarrow S^{4}$ be constrained Willmore so that

$$
d^{\nabla}(* A+\eta)=0 \quad \text { with } \quad A \not \equiv 0
$$

and let $\alpha \in V^{*}$ satisfying $V=L \oplus \operatorname{ker} \alpha$. Then the Bäcklund transform $\tilde{f}: \tilde{M} \rightarrow S^{4}$ of $f$ obtained by integration of the linear system $H_{K L}=\{(* A+\eta) b \mid b \in \operatorname{ker} \alpha\}$ is constrained Willmore, provided $\tilde{f}$ is a Frenet curve. 
Proof. To simplify notations, we assume that $\tilde{f}$ is defined on $M$. As before we define

$$
\Omega=* A+\eta: V \rightarrow H^{0}(K L)
$$

and we denote by $\tilde{H}$ the 2 -dimensional linear system obtained by integration of $\{\Omega b \mid b \in$ ker $\alpha$ \}. Moreover, let $\tilde{\nabla}$ be the flat connection on $\tilde{V}=\tilde{H}^{*}$ and $\psi \in \Gamma(L)$ with $\langle\alpha, \psi\rangle=1$. Consider the bundle map

$$
T: \operatorname{ker} \alpha \rightarrow \tilde{H} / \psi \mathbb{H},
$$

with $\hat{\nabla} T=\Omega$ where $\hat{\nabla}$ is the connection on $\operatorname{Hom}(\operatorname{ker} \alpha, \tilde{H} / \psi \mathbb{H})$ induced by the trivial connection $\nabla$ on $\operatorname{ker} \alpha$ and by the flat connection $\nabla^{L}$ on $\tilde{H} / \psi \mathbb{H}$. The latter connection is well-defined since $\nabla^{L} \psi=0$ and $\tilde{H} \subset H^{0}(L) \subset \Gamma(L)$. Let

$$
T^{*}: \operatorname{ker} \psi=(\tilde{H} / \psi \mathbb{H})^{-1} \rightarrow H / \alpha \mathbb{H}=(\operatorname{ker} \alpha)^{-1}
$$

be the dual map of $T$. From (3) we see that $\nabla^{L^{-1}} \beta \in H^{0}\left(K L^{-1}\right)$ for any $\beta \in H^{0}\left(L^{-1}\right)$ and therefore

$$
\hat{\nabla} T^{*}: \operatorname{ker} \psi \rightarrow H^{0}\left(K L^{-1}\right),
$$

where $\hat{\nabla}$ is the connection induced by the trivial connection $\tilde{\nabla}$ on $\operatorname{ker} \psi$ and $\nabla^{L^{-1}}$ on $H / \alpha \mathbb{H}$. Let $\tilde{S}$ be the mean curvature sphere congruence of $\tilde{f}$ and let $\tilde{A}$ be as usual defined by (14). Using the splitting $\tilde{V}=\operatorname{ker} \psi \oplus L^{-1}$, we define the linear map $\tilde{\Omega}: \tilde{V} \rightarrow \Gamma\left(K L^{-1}\right)$ by

$$
\left.\tilde{\Omega}\right|_{L^{-1}}=\left.* \tilde{A}\right|_{L^{-1}} \quad \text { and }\left.\quad \tilde{\Omega}\right|_{\operatorname{ker} \psi}=\hat{\nabla} T^{*} .
$$

We show that $\tilde{\Omega}$ is $d^{\tilde{\nabla}}$-closed and differs from $* \tilde{A}$ by a 1 -form $\tilde{\eta} \in \Gamma\left(K \tilde{\mathcal{R}}_{+}\right)$where

$$
\tilde{\mathcal{R}}=\operatorname{Hom}\left(\tilde{V} / L^{-1}, L^{-1}\right) .
$$

Since $T$ and $T^{*}$ are parallel with respect to the connections $\nabla$ on $V$ and $\tilde{\nabla}$ on $\tilde{V}$, we get

$$
\langle\psi, \tilde{\Omega} \wedge \tilde{\nabla} \alpha\rangle=\left\langle\psi, \hat{\nabla} T^{*} \wedge \tilde{\nabla} \alpha\right\rangle=-\langle\hat{\nabla} T \wedge \nabla \psi, \alpha\rangle=-\langle\Omega \wedge \nabla \psi, \alpha\rangle,
$$

where we also used that $\tilde{\nabla} \alpha \in \Omega^{1}(\operatorname{ker} \psi)$. Lemma A.1 and Lemma A.2 below show that

$$
\langle\psi, \tilde{\Omega} \wedge \tilde{\nabla} \alpha\rangle=-d\langle\psi, \tilde{S} \tilde{\nabla} \alpha\rangle
$$

and Lemma A.3 then implies that

$$
\tilde{\eta}=\tilde{\Omega}-* \tilde{A} \in \Gamma\left(K \tilde{\mathcal{R}}_{+}\right) .
$$

In particular, $\tilde{\eta} \wedge \tilde{\delta}=0$, and $\left.\tilde{\eta}\right|_{L^{-1}}=0$ so that

$$
\left.d^{\tilde{\nabla}}(* \tilde{A}+\tilde{\eta})\right|_{L^{-1}}=\left.d^{\tilde{\nabla}} * \tilde{A}\right|_{L^{-1}}+\tilde{\eta} \wedge \tilde{\delta}=0 .
$$

Here $\left.d^{\tilde{\nabla}} * \tilde{A}\right|_{L^{-1}}=0$ holds by type considerations for canonical complex structures of Frenet curves, see [LP03].

Using the splitting $\tilde{V}=L^{-1} \oplus \operatorname{ker} \psi$, we decompose $\left.\tilde{\nabla}\right|_{L^{-1}}=\nabla^{L^{-1}}+\tilde{\delta}$. By (2) and (3) we get for $\tilde{b} \in \operatorname{ker} \psi$

$$
\left(d^{\tilde{\nabla}}(* \tilde{A}+\tilde{\eta})\right) \tilde{b}=d^{\tilde{\nabla}}(\tilde{\Omega} \tilde{b})=d^{\nabla^{L^{-1}}}\left(\nabla^{L^{-1}}\left(T^{*} \tilde{b}\right)\right)=0 .
$$

Thus, $\tilde{f}$ is constrained Willmore since $d^{\tilde{\nabla}}(* \tilde{A}+\tilde{\eta})=0$ on $\tilde{V}=\operatorname{ker} \psi \oplus L^{-1}$ by (18) and (19).

As a corollary we show that the Bäcklund transformation also preserves Willmore surfaces in $S^{4}$. 
Corollary 5.4 ([BFL $\left.\left.{ }^{+} 02\right]\right)$. Let $f: M \rightarrow S^{4}$ be Willmore and $\alpha \in V^{*}$ so that $V=$ $L \oplus \operatorname{ker} \alpha$. Then the Bäcklund transform $\tilde{f}: \tilde{M} \rightarrow S^{4}$ given by integration of the linear system $H_{K L}=\{* A b \mid b \in \operatorname{ker} \alpha\}$ is Willmore provided $\tilde{f}$ is Frenet.

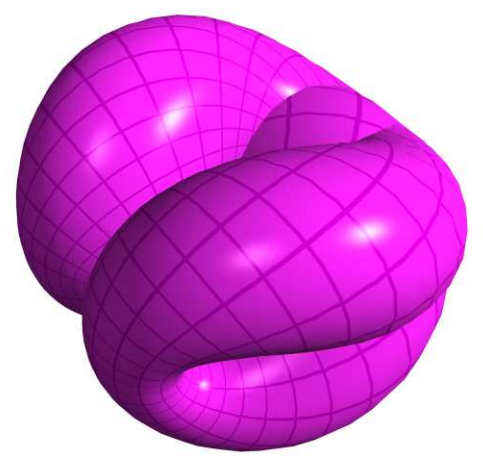

Fig. 2. Willmore sphere in $\mathbb{R}^{3}$ obtained by Bäcklund transformation, [BP], [Hel02]

Proof. In view of Theorem 5.3 we have to show that the (1,0)-form $\tilde{\eta}$ in (17) vanishes. Let $\tilde{H}$ be the linear system obtained by integration of $H_{K L}$ and $\tilde{\nabla}$ the flat connection on $\tilde{V}=\tilde{H}^{*}$. Let $\varphi \in \tilde{V}^{*}$ with $\nabla^{L} \varphi=* A b$. Since $\tilde{\nabla} \varphi=0$ we get $d\langle\varphi, \alpha\rangle=\langle\varphi, \tilde{\nabla} \alpha\rangle$. On the other hand $\nabla^{L^{-1}} \alpha=0$ implies $d\langle\varphi, \alpha\rangle=\left\langle\nabla^{L} \varphi, \alpha\right\rangle=\langle * A b, \alpha\rangle$, which shows that

$$
\tilde{\nabla} \alpha=\tilde{b}\langle * A b, \alpha\rangle
$$

where $\tilde{b} \in \operatorname{ker} \psi$ is given by $\langle\varphi, \tilde{b}\rangle=1$. Similarly, if we choose $\beta \in V^{*}$ with $\langle\beta, b\rangle=1$ then $T^{*} \tilde{b}=\beta$ and $\langle\tilde{\Omega} \tilde{b}, \psi\rangle=\left\langle\nabla^{L^{-1}} \beta, \psi\right\rangle=\langle\beta, \nabla \psi\rangle$. Therefore,

$$
\nabla \psi=b\langle\tilde{\Omega} \tilde{b}, \psi\rangle \text {. }
$$

By Lemma A.4 below we have

$$
\langle\alpha, A \nabla \psi\rangle=-\overline{\langle\psi, * \tilde{A} * \tilde{\nabla} \alpha\rangle},
$$

and, plugging (20) and (21) into this equation, we get

$$
\langle\psi, \tilde{\Omega} \tilde{b}\rangle=\langle\psi, * \tilde{A} \tilde{b}\rangle .
$$

Therefore $\tilde{\Omega} \tilde{b}=* \tilde{A} \tilde{b}$ and $\tilde{\eta}=0$, which shows that $\tilde{f}$ is Willmore.

A. Appendix. We conclude the paper by providing the technical lemmas used in the proofs of Theorem 5.3 and Corollary 5.4. Since all calculations are done locally, we assume that all appearing curves are Frenet curves on the Riemann surface $M$.

Lemma A.1. Let $f: M \rightarrow \mathbb{H P}^{n}$ be a Frenet curve with Bäcklund transform

$$
\tilde{f}=B_{\alpha, \tilde{H}}(f): M \rightarrow \mathbb{H P}^{k}
$$

with respect to $\alpha \in H^{0}\left(L^{-1}\right)$, where $\tilde{H}$ is a linear system obtained by integration of a $k$ dimensional linear system $H_{K L} \subset H^{0}(K L)$. Let $\psi \in \Gamma(L)$ be the section with $\langle\alpha, \psi\rangle=1$. Then the canonical complex structures $S$ of $f$ and $\tilde{S}$ of $\tilde{f}$ satisfy

$$
-\overline{d\langle\alpha, S \nabla \psi\rangle}=d\langle\psi, \tilde{S} \tilde{\nabla} \alpha\rangle .
$$


Proof. Note that the evaluation pairing on $\tilde{H} \subset H^{0}(L)$ satisfies $\langle\tilde{\psi}, \tilde{\alpha}\rangle=\overline{\langle\tilde{\alpha}, \tilde{\psi}\rangle}$ for $\tilde{\psi} \in \Gamma(L)$ and $\tilde{\alpha} \in \Gamma\left(L^{-1}\right)$. The complex structure on $L^{-1}$ is the dual of the complex structure on $L$ so that

$$
\langle\alpha, S \psi\rangle=-\langle\psi, \tilde{S} \alpha\rangle
$$

Since $\psi \in \Gamma(L) \subset \Gamma\left(V_{n-1}\right)$, we get by (14) and (12)

$$
d\langle\alpha, S \psi\rangle=\langle\alpha,(\nabla S) \psi+S \nabla \psi\rangle=\langle\alpha,-2 * A \psi+S \nabla \psi\rangle .
$$

On the other hand, since $* \delta=S \delta$, we see

$$
* \nabla \psi=S \nabla \psi-\psi\langle\alpha, S \nabla \psi\rangle,
$$

and calculate

$$
\begin{aligned}
S \psi * d\langle\alpha, S \psi\rangle & =S \psi\langle\alpha,(* \nabla S) \psi+S * \nabla \psi\rangle \\
& =S \psi\langle\alpha, 2 A \psi-\nabla \psi-S \psi\langle\alpha, S \nabla \psi\rangle\rangle \\
& =S \psi\langle\alpha, 2 A \psi-S \psi\langle\alpha, S \nabla \psi\rangle\rangle \\
& =\psi\langle\alpha, 2 * A \psi+\psi\langle\alpha, S \nabla \psi\rangle\rangle,
\end{aligned}
$$

where we used that $\langle\alpha, \nabla \psi\rangle=0$ and $S \psi\langle\alpha, \chi\rangle=\psi\langle\alpha, S \chi\rangle$ for $\chi \in \Gamma(L)$. Combining the above computations, we get

$$
\psi d\langle\alpha, S \psi\rangle+S \psi * d\langle\alpha, S \psi\rangle=2 \psi\langle\alpha, S \nabla \psi\rangle
$$

and therefore

$$
2\langle\alpha, S \nabla \psi\rangle=d\langle\alpha, S \psi\rangle+\langle\alpha, S \psi\rangle * d\langle\alpha, S \psi\rangle
$$

This implies

$$
\langle\alpha, S \nabla \psi\rangle \in \operatorname{im} \mathbb{H}
$$

since $\langle\alpha, S \psi\rangle^{2}=-1$ and thus $\overline{\langle\alpha, S \psi\rangle * d\langle\alpha, S \psi\rangle}=-\langle\alpha, S \psi\rangle * d\langle\alpha, S \psi\rangle$. Using (23) we compute

$$
\begin{aligned}
d\langle\alpha, S \psi\rangle+\langle\alpha, S \psi\rangle * d\langle\alpha, S \psi\rangle & =-d\langle\psi, \tilde{S} \alpha\rangle+\langle\psi, \tilde{S} \alpha\rangle * d\langle\psi, \tilde{S} \alpha\rangle \\
& =-2 d\langle\psi, \tilde{S} \alpha\rangle+2\langle\psi, \tilde{S} \tilde{\nabla} \alpha\rangle
\end{aligned}
$$

and conclude

$$
\overline{\langle\alpha, S \nabla \psi\rangle}=-\langle\alpha, S \nabla \psi\rangle=d\langle\psi, \tilde{S} \alpha\rangle-\langle\psi, \tilde{S} \tilde{\nabla} \alpha\rangle .
$$

The assertion (22) follows by differentiating this equation.

Lemma A.2. Let $f: M \rightarrow \mathbb{H}^{n}$ be a Frenet curve with canonical complex structure $S$ and $\eta \in \Gamma\left(K \mathcal{R}_{+}\right)$. Then

$$
-2\left\langle\alpha,(* A+\eta) \wedge \nabla \tilde{\psi}_{n-1}\right\rangle=d\left\langle\alpha, S \nabla \psi_{n-1}\right\rangle .
$$

for all $\psi_{n-1} \in \Gamma\left(V_{n-1}\right)$.

Proof. For $\psi_{n-1} \in \Gamma\left(V_{n-1}\right)$ we see $* Q \wedge \nabla \psi_{n-1}=* Q \wedge \delta_{n-1} \psi_{n-1}=0$ by type. Since $\nabla S=2(* Q-* A)$ we get

$$
d\left\langle\alpha, S \nabla \psi_{n-1}\right\rangle=-2\left\langle\alpha, * A \wedge \nabla \psi_{n-1}\right\rangle .
$$

Moreover, $\eta \wedge \nabla \psi_{n-1}=\eta \wedge \delta_{n-1} \psi_{n-1}=0$ since $\left.\eta\right|_{V_{n-1}}=0$ and $* \eta=\eta S$. 
Lemma A.3. Let $f: M \rightarrow \mathbb{H} \mathbb{P}^{n}$ be a Frenet curve with canonical complex structure $S$, $\alpha \in V^{*}$ with $L \oplus \operatorname{ker} \alpha=V$, and assume that $\eta: V \rightarrow \Gamma(K L)$ satisfies $\left.\eta\right|_{V_{n-1}}=0$ and

$$
-2\left\langle\alpha,(* A+\eta) \wedge \nabla \psi_{n-1}\right\rangle=d\left\langle\alpha, S \nabla \psi_{n-1}\right\rangle
$$

for all $\psi_{n-1} \in \Gamma\left(V_{n-1}\right)$. Then $\eta \in \Gamma\left(K \mathcal{R}_{+}\right)$.

Proof. Combining (26) with the assumption (27), we get $\left\langle\alpha, \eta \wedge \nabla \psi_{n-1}\right\rangle=0$. Since $\eta$ has values in $L$ and $L \oplus \operatorname{ker} \alpha=V$, we see $0=\eta \wedge \nabla \psi_{n-1}=\eta \wedge \delta_{n-1} \psi_{n-1}$, where we also used $\left.\eta\right|_{V_{n-1}}=0$. Since $\delta_{n-1} \psi_{n-1} \neq 0$ this implies either $\operatorname{Im} \delta_{n-1} \subset \operatorname{ker} \eta$, i.e. $\eta \equiv 0$, or $* \eta=\eta S$. In both cases $\eta \in \Gamma\left(K \mathcal{R}_{+}\right)$.

Lemma A.4. Let $f: M \rightarrow \mathbb{H}^{n}$ be a Frenet curve with Bäcklund transform

$$
\tilde{f}=B_{\alpha, \tilde{H}}(f): M \rightarrow \mathbb{H}^{k}
$$

with respect to $\alpha \in H^{0}\left(L^{-1}\right)$, where $\tilde{H}$ is a linear system obtained by integration of a $k$ dimensional linear system $H_{K L} \subset H^{0}(K L)$. Let $\psi \in \Gamma(L)$ be the section with $\langle\alpha, \psi\rangle=1$. Then the canonical complex structures $S$ of $f$ and $\tilde{S}$ of $\tilde{f}$ satisfy

$$
\langle\alpha, A \nabla \psi\rangle=-\overline{\langle\psi, * \tilde{A} * \tilde{\nabla} \alpha\rangle} .
$$

Proof. By Lemma A.1 and Lemma A.2 we see that

$$
-\overline{\langle\alpha, * A \wedge \nabla \psi\rangle}=\langle\psi, * \tilde{A} \wedge \tilde{\nabla} \alpha\rangle .
$$

With (24) and $A=* A S$ we compute

$$
\langle\alpha, * A \wedge \nabla \psi\rangle=\langle\alpha, * A * \nabla \psi+A \nabla \psi\rangle=\langle\alpha, 2 * A * \nabla \psi\rangle+\langle\alpha, * A \psi\rangle\langle\alpha, S \nabla \psi\rangle,
$$

and similarly

$$
\langle\psi, * \tilde{A} \wedge \tilde{\nabla} \alpha\rangle=\langle\psi, 2 \tilde{A} \tilde{\nabla} \alpha\rangle-\langle\psi, * \tilde{A} \alpha\rangle\langle\psi, \tilde{S} \tilde{\nabla} \alpha\rangle .
$$

But this implies (28) since

$$
\overline{\langle\alpha, * A \psi\rangle\langle\alpha, S \nabla \psi\rangle}=\langle\psi, * \tilde{A} \alpha\rangle\langle\psi, \tilde{S} \tilde{\nabla} \alpha\rangle
$$

by using (25)

$$
-2\langle\alpha, * A \psi\rangle=\langle\alpha,(\nabla S) \psi\rangle=d\langle\alpha, S \psi\rangle-\langle\alpha, S \nabla \psi\rangle=-\langle\psi, \tilde{S} \tilde{\nabla} \alpha\rangle .
$$

and similarly $2\langle\psi, * \tilde{A} \alpha\rangle=\langle\alpha, S \nabla \psi\rangle$.

\section{References}

$\left[\mathrm{BFL}^{+} 02\right] \quad$ F. Burstall, D. Ferus, K. Leschke, F. Pedit, and U. Pinkall, Conformal Geometry of Surfaces in $S^{4}$ and Quaternions, Lecture Notes in Mathematics, Springer, Berlin, 2002.

[Bia80] L. Bianchi, Ueber die Flächen mit constanter negativer Krümmung, Math. Ann. 16 (1880), 577-582.

[BP] C. Bohle and P. Peters, Soliton spheres, in preparation.

[Dar99] G. Darboux, Sur la déformation des surfaces du second degré, Comptes Rendus 128 (1899), 1299-1305. 
[FLPP01] D. Ferus, K. Leschke, F. Pedit, and U. Pinkall, Quaternionic holomorphic geometry: Plücker formula, Dirac eigenvalue estimates and energy estimates of harmonic 2tori, Invent. Math. 146 (2001), 507-593.

[Hel02] U. Heller. Construction, transformation, and visualization of Willmore surfaces, $\mathrm{PhD}$ thesis, University of Massachusetts, 2002.

[LP03] K. Leschke and F. Pedit, Osculates and envelopes of Willmore surfaces, preprint at http://arXiv.org/math.DG/0306150, 2003.

[Pet04] P. Peters, Soliton spheres, PhD thesis, Technische Universität Berlin, 2004.

[PP98] F. Pedit and U. Pinkall, Quaternionic analysis on Riemann surfaces and differential geometry, Doc. Math. J. DMV, Extra Volume ICM, Vol. II, 1998, 389-400. 